\title{
Attenuation effects on imaging diagnostics of hollow-cone sprays
}

\author{
Volker Sick and Boris Stojkovic
}

\begin{abstract}
Detrimental effects to quantitative interpretation of Mie and laser-induced fluorescence images of hollowcone sprays were investigated. The attenuation of the laser beam leads to locally unknown intensities rendering it impossible to obtain high-fidelity images of these sprays. Two strategies that use bidirectional illumination of the spray are discussed and evaluated. Conditions for which a bidirectional illumination, single-image detection will allow good recovery of the spray structure are identified. Furthermore, the attenuation of laser-induced fluorescence signals on their path through the spray is quantified. (C) 2001 Optical Society of America

OCIS codes: $\quad 100.3010,290.5820,290.2200,110.2960,260.2510,999.9999$.
\end{abstract}

\section{Introduction}

With the increased interest in gasoline direct injection as a possible major improvement toward cleaner automobile engines, the demand for the application of laser diagnostic techniques has greatly increased in recent years. ${ }^{1}$ Optical measurements in engines require a careful examination of the optical properties of all processes and equipment involved, in particular when the measurement of absolute quantities is required. It has been shown in engine experiments that laser beam ${ }^{2}$ and signal ${ }^{3,4}$ attenuations have a significant influence, eventually perturbing or preventing investigations of extended areas within the cylinder or leading to significant misinterpretation of signal intensities. Many of the frequently used laser techniques rely on excitation and detection in the UV spectral region where absorption by transient combustion products such as polycyclic hydrocarbons is strong even at small concentrations. Even before combustion starts, these effects play an important role. Among the most important quantities to be measured are local fuel concentrations and the fuelair mixing process. Injectors that produce hollowcone sprays are commonly used to introduce the fuel directly into the combustion chamber. Initially, the

V. Sick (vsick@umich.edu) and B. Stojkovic are with the Department of Mechanical Engineering, University of Michigan, Ann Arbor, Michigan 48109-2121.

Received 15 August 2000; revised manuscript received 31 January 2001.

0003-6935/01/152435-08\$15.00/0

(C) 2001 Optical Society of America fuel is a liquid sheet that begins to break up into droplets and evaporates along its path into the cylinder.

When we study the local fuel distribution with laser-based imaging techniques, the high density of the fuel droplets leads to substantial attenuation of the exciting laser beam and the induced signals. Bidirectional excitation schemes have been used previously to address the attenuation of the exciting laser light.5,6 These approaches used two light sheets to illuminate the spray from opposite sides in an attempt to overcome at least some of the loss of laser light. Detection of the signals excited by both beams is performed simultaneously, thus effectively leading to a signal that is proportional to the sum of the local laser intensities of the two beams. Su et al. ${ }^{6}$ also report a technique that uses two-dimensional Miescattering images to gain information about the local attenuation of laser intensity under the assumption that absorption is negligible. An iterative correction algorithm was developed by Hertz and Alden ${ }^{7}$ to correct laser-induced fluorescence (LIF) measurements for attenuation effects. This requires the spatially resolved measurement of the fluorescence signals and the absolute attenuation across the flame. Versluis et al. ${ }^{8}$ described a bidirectional excitation method to measure absolute concentrations of hydroxyl radicals in flames using LIF. The purpose of their research was to eliminate the influence of fluorescence quenching and the need for separate calibration to absolute concentration. In contrast with the other techniques, the signals excited by the two laser beams have to be detected separately and can then be processed. This will allow for only single- 
pulse measurements, if two time-separated laser beams and two cameras are used. The same holds for an approach that was described by Talley et al. ${ }^{9}$ After they measured LIF signals with excitation from counterpropagating sheets, a numerical procedure was used to recover the true intensity distribution. The underlying assumption was that only the laser beam, but not the signals, was attenuated.

However, in addition to distortion of measured spray images by attenuation of the exciting laser light, the images can suffer further from nonuniform attenuation of the signal. As reported by Hildenbrand et al. ${ }^{4}$ and Knapp et al., ${ }^{3}$ the attenuation can be wavelength dependent. Investigations of the effect of signal loss that is due to scattering or absorption therefore need to be carried out with a suitable wavelength resolution.

We studied and quantified the attenuation of exciting laser beams and Mie, as well as LIF, signals for a hollow-cone spray with a $\mathrm{KrF}$ excimer laser at 248 $\mathrm{nm}$ as the light source. We discuss possible ways for correction and their limitations.

\section{Theoretical Background}

\section{A. Laser Beam Attenuation}

In the case of Mie scattering or LIF in the linear regime, the measured signals will be proportional to the number density $N$ of particles (droplets or molecules, respectively) and the laser intensity $I_{\text {laser }}$ at the location of the measurement:

$$
I_{\text {signal }}=N I_{\text {laser }} \sigma C,
$$

where $\sigma$ denotes a scattering cross section or absorption coefficient and $C$ includes information about detection efficiency of the equipment as well as fluorescence quantum yield where appropriate. Two mechanisms exist to attenuate the intensity of a laser beam on its path along an $x$ axis through a spray. Absorption on a molecular level and scattering of light from the surface of the droplets can have varying degrees of contribution to the overall attenuation. In practical sprays, this attenuation often exceeds tolerable levels of a few percent; and, as such, the measured distribution of, for example, Mie scattering or LIF signals will not reflect the true distribution of the local fuel concentration. Before the measurements obtained under such conditions are interpreted, the measured signals must be referenced to the local laser intensity. Because the local laser intensity at a position $x$ cannot be measured directly, other means have to be found to correct the measured signals for changes in the local laser intensity. This can be addressed by use of a counterpropagating laser beam arrangement in which the spray is illuminated with two light sheets introduced from opposite sides of the spray.

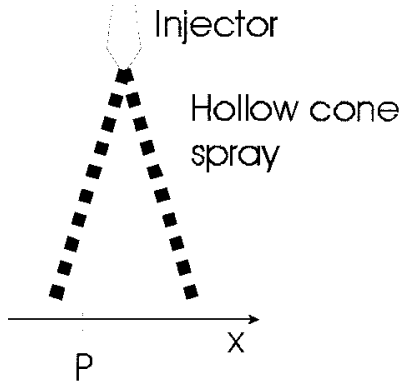

Fig. 1. Schematic view of a vertical cross section through a hollow-cone spray and nomenclature for the coordinate system and laser beam direction.

The local laser intensity at position $P$ (Fig. 1) for the forward-traveling laser beam is determined by

$$
I_{\text {forward }}(P)=I_{0, \text { forward }} \exp \left[-\int_{0}^{P} \sigma N(x) \mathrm{d} x\right] .
$$

Similarly, the laser intensity at position $P$ introduced by the backward laser beam is given by

$$
I_{\text {backward }}(P)=I_{0, \text { backward }} \exp \left[-\int_{P}^{L} \sigma N(x) \mathrm{d} x\right] \text {. }
$$

This description assumes use of a parallel, top-hat light sheet as was used for the experiments in this study. For the case in which both beams illuminate the spray simultaneously, the total measured signal at location $P$ will be the sum of two contributions:

$$
\begin{aligned}
I_{\text {signal }}(P)= & {\left[I_{\text {forward laser }}(P) N(P)\right.} \\
& \left.+I_{\text {backward laser }}(P) N(P)\right] \sigma C .
\end{aligned}
$$

We show in Section 4 if and how this procedure will be suitable for complete correction of attenuation with the spray.

A second method also uses counterpropagating illumination but with a sequential recording of two images. The first image is recorded during illumination by a forward light sheet, and the second image is recorded during a second, slightly time-delayed laser pulse. The images can then be processed in a way that was introduced by Versluis et al. ${ }^{8}$ for quantitative measurements of hydroxyl radicals in flames.

Because the measured signals are proportional to the local laser intensity, the ratio of the measured signals induced by the two beams can be written as

$$
R(P)=\frac{I_{\text {signal forward }}(P)}{I_{\text {signal backward }}(P)}=\frac{I_{\text {forward }}(P)}{I_{\text {backward }}(P)} .
$$

We can rewrite the natural logarithm of this expression using Eqs. (2) and (3) to yield

$$
\begin{aligned}
\ln R(P)= & \ln \left(\frac{I_{0, \text { forward }}}{I_{0, \text { backward }}}\right)-\int_{0}^{P} \sigma N(P) \mathrm{d} x \\
& +\int_{P}^{L} \sigma N(P) \mathrm{d} x .
\end{aligned}
$$


By moving the limits of integration such that the last term is integrated over the entire path through the spray, we obtain an expression in which only the first integral remains a function of $x$ :

$$
\begin{aligned}
\ln R(P)= & \ln \left(\frac{I_{0, \text { forward }}}{I_{0, \text { backward }}}\right)-2 \int_{0}^{P} \sigma N(P) \mathrm{d} x \\
& +\int_{0}^{L} \sigma N(P) \mathrm{d} x .
\end{aligned}
$$

Because the ratio of the initial laser intensities is constant, the spatial derivative of Eq. (7) yields

$$
\frac{\mathrm{d}}{\mathrm{d} x} \ln R(P)=2 \sigma N(P) .
$$

The local number density is then evaluated as

$$
N(P)=\frac{1}{2 \sigma} \frac{\mathrm{d}}{\mathrm{d} x} \ln R(P) .
$$

This result shows that the measurement of the number density is independent of the ratio of the initial laser intensities, a fact that simplifies the experimental research.

\section{B. Signal Attenuation}

The signals can be diminished in the same way as the exciting laser beam is attenuated by scattering and absorption. To study this effect, the wavelength of the signal must be considered. For information on attenuation of Mie-scattering signals, the overall attenuation of the exciting beam can provide a first step toward a correction for measured signals. This will differ for LIF signals. To investigate the attenuation of LIF signals with a spray, an experiment as described below was designed for the case in which the fuel is a mixture of 3-pentanone as a fluorescence tracer in nonfluorescing isooctane. This is a possible mixture for optical engine studies addressing fuel mixing and temperatures. ${ }^{10,11}$ For the particular mixture under study, the absorption and emission bands of the tracer do not overlap. Also, no other absorbers are present in the fuel-tracer mixture. Any attenuation therefore will be due solely to scattering effects because self-absorption cannot take place. This avoids problems that occur when absorbers trap fluorescence as is discussed for hydroxyl radicals, for example, by Quagliaroli et al. ${ }^{12}$ In general, a measurement should be performed with light at the same spectral intensity distribution as the fluorescence light so that possible fluorescence trapping, which could occur by the absorption by molecules other than the emitters, will also be included in the measurement. A setup for this was realized by use of LIF signals created in a laser-illuminated cuvette filled with a mixture of 3-pentanone and isooctane as the light source to measure the spatially resolved attenuation of fluorescence signals by sprays.

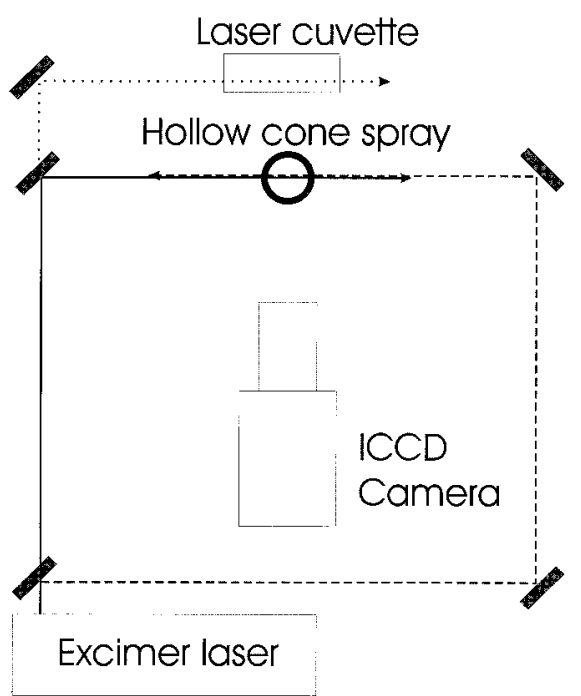

Fig. 2. Experimental setup for counterpropagating excitation to study hollow-cone sprays with Mie scattering and LIF. The dotted laser beam illustrates the setup to measure the signal attenuation across the spray structure. ICCD, intensified CCD.

\section{Experiment}

Experiments were carried out in a static enginelike spray test rig. A hollow-cone injector (Zexel) with a nominal spray angle of $30^{\circ}$ was used. The tracerfuel mixture (0.1\% 3-pentanone in isooctane) was delivered to the injector at a pressure of $6.5 \mathrm{MPa}$. The injector was opened for $1.2 \mathrm{~ms}$ during which time the fuel was injected into an open-atmosphere chamber. Laser light of $248 \mathrm{~nm}$ was produced with a $\mathrm{KrF}$ excimer laser (Lambda Physik EMG 150 TMSC). The beam was split close to equal intensities, and the two beams formed two vertical light sheets with cylindrical lenses. The beams intersected the spray symmetrically in its center plane. Laser intensities were kept at $5 \mathrm{MW} / \mathrm{cm}^{2}$, a value that was found to ensure that the LIF signals would respond linearly to the pulse energy. The energy for each laser pulse was measured with a photodiode (LaVision) and recorded by a sample\&hold board (LaVision) before storing the information directly with the measured Mie-scattering and LIF image. The signals were detected with an intensified CCD camera with 14-bit resolution and $576 \times 384$ pixels (LaVision FlameStar II). Blocking either the forward or the backward beam was the method we used to measure averaged fluorescence and Mie-scattering images of the spray. A new beam-splitter and filter setup was used to separate fluorescence from Mie scattering with high overall throughput. ${ }^{13}$ This setup allows the simultaneous detection of both signals with only one CCD camera. Figure 2 illustrates the setup.

To investigate the attenuation of fluorescence signals, as viewed from the camera, a dye laser cuvette was filled with a dilute 3-pentanone-isooctane mixture that was placed behind the spray (see Fig. 2). Only one of the laser sheets was used for this measurement. The sheet was aligned through the cell, 


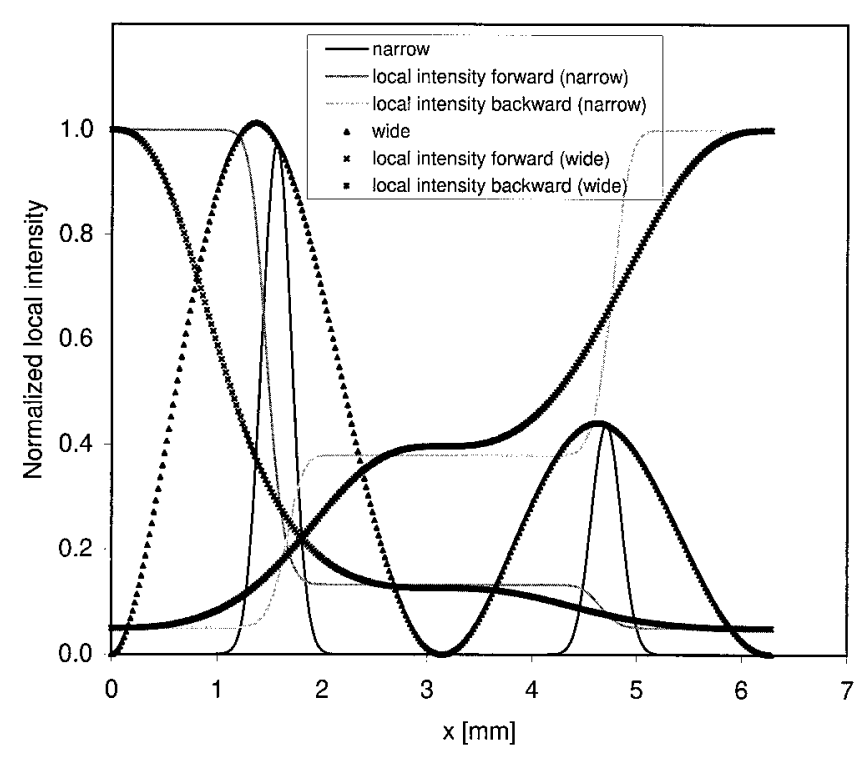

Fig. 3. Assumed model fuel distribution along a horizontal cross section through the hollow-cone spray. Two cases that are considered are denoted as narrow and wide fuel distribution. The local laser intensities calculated for the forward and backward laser beams are given for an overall attenuation of $95 \%$.

exciting the 3-pentanone to fluoresce. When we used the same tracer as for the spray, the emission had the same spectral intensity distribution as signals generated in the spray itself. When recording an image without the spray, we measured a baseline for the attenuation experiment. This baseline includes all spatial intensity variations and the filter function for the fluorescence. After recording this reference image as an average of 15 individual exposures, we repeated the measurement with the spray. After the start of fuel injection, single-shot and averaged images taken at the same delays were recorded. As a result, the ratio of the images represents the spatial variation of signal attenuation.

\section{Results and Discussion}

\section{A. Laser Beam Attenuation}

To study the advantages and disadvantages of the two approaches for reconstruction, we conducted a numerical analysis before reducing the experimental data from the sprays. A model function for a horizontal cross section through a hollow-cone spray was chosen $\operatorname{as}^{n} \sin ^{n}(x)$. For $n=50$, a realistic half-width for the dense spray region of approximately $350 \mu \mathrm{m}$ is obtained with a separation between the two legs of $3.1 \mathrm{~mm}$ as shown in Fig. 3. For cases with a broader fuel distribution, we chose the exponent $n=2$, which yields a half-width of $1.6 \mathrm{~mm}$. The number of data points was set to 300 to resemble the number of pixels on frequently used CCD cameras. This corresponds to approximately $20 \mu \mathrm{m}$ imaged onto one pixel. These data were used to evaluate the performance of the reconstruction procedures before we applied them to measured data from a real spray. A variable total attenuation of the laser beam intensity across the
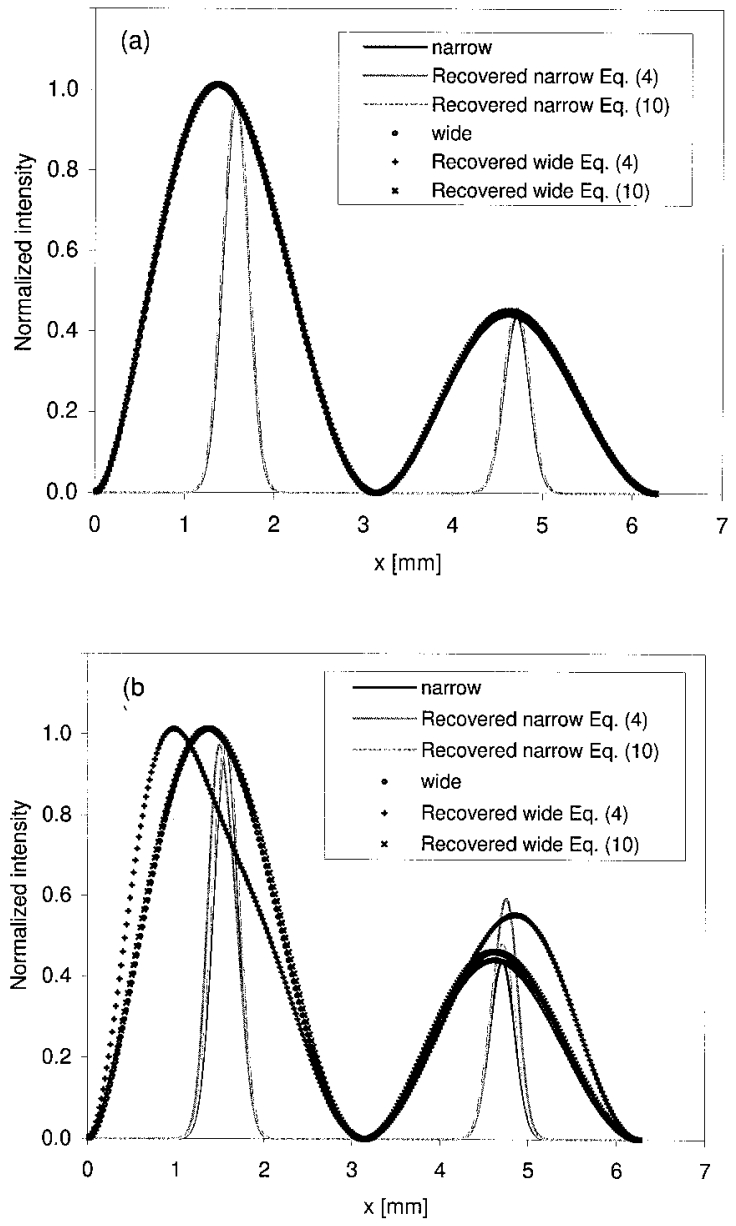

Fig. 4. Reconstructed fuel distribution: (a) $50 \%$ overall attenuation and (b) $95 \%$ overall attenuation. Both reconstruction strategies are shown for comparison.

spray was selected for the evaluation. Figure 3 shows the local intensities for an overall attenuation of $95 \%$. A linear gradient from left to right was superimposed to mimic possible asymmetric fuel distributions that can occur in real sprays. In view of the ratio of two signals that has to be formed according to Eq. (5), an offset of $0.01 \%$ of the peak signal was added to the assumed distribution. Evaluation of real data will have to use a similar offset to avoid division by zero errors. This will not affect the quantitative nature of the measurement, because $0.01 \%$ of the 14-bit resolution of frequently used CCD cameras for these studies corresponds to less than two counts.

We calculated the local laser intensity for the forward and backward beams assuming equal initial intensity for both beams (see Fig. 4). With the local intensities obtained, the expected signal induced by each beam can be calculated for each position. In view of Eq. (4), the individual intensities are added. The analysis of the same data, by use of the derivative according to Eq. (9), poses the question as to which method should be used to calculate the derivative. Although the derivative of the sine function used for the model fuel distribution can be deter- 

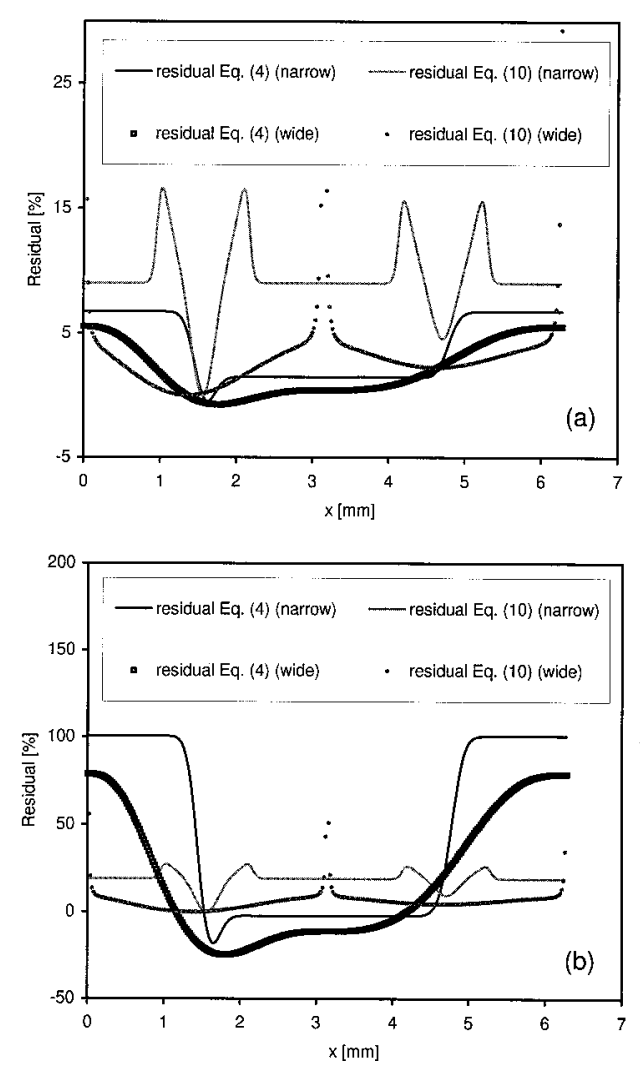

Fig. 5. Observed deviation between true fuel distribution and reconstructed profiles. Results shown are for both reconstruction methods and fuel distributions. (a) $50 \%$ overall attenuation and (b) $95 \%$ overall attenuation.

mined analytically, this will not be possible with an arbitrary, and possibly noisy, measured signal. Calculation of forward-backward differences is a simple and frequently used approach to determine the derivative for signal images. ${ }^{14}$ At a point $x_{n}$ the derivative is determined as

$$
\frac{\mathrm{d}}{\mathrm{d} x}\left[f\left(x_{n}\right)\right]=\frac{f\left(x_{n+1}\right)-f\left(x_{n-1}\right)}{2 \Delta x} .
$$

This calculation can be performed quickly and easily. Given that the spatial resolution of CCD cameras is quite limited, significant errors for the derivative in regions where the gradients change rapidly, i.e., the region near the peaks of the assumed fuel distribution, would be expected.

Reconstructed distributions for $50 \%$ and $95 \%$ overall attenuation are shown in Figs. 4(a) and 4(b). All profiles were normalized to unity at their highest intensity. Deviations are discussed as a percent difference with respect to the original intensity level. In the case of $50 \%$ attenuation, both reconstruction methods recover the structure and intensity ratio of the two peaks within acceptable error limits. Neither the narrow nor the wide distribution is spatially overly distorted. Local deviations are shown in Fig. 5. Comparison between the original and the recovered profiles is even more favorable when we consider

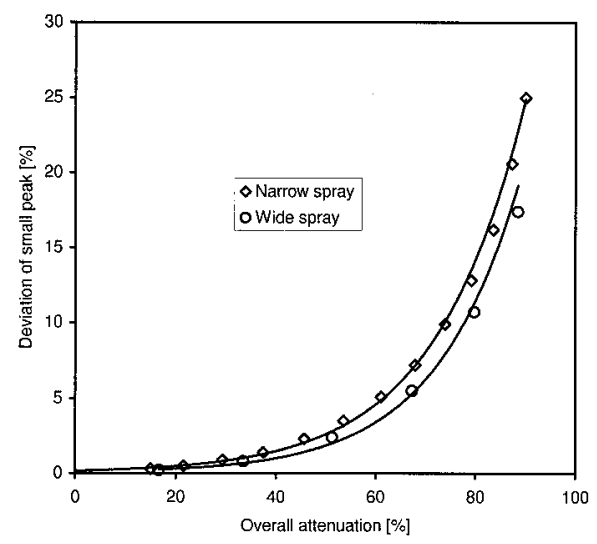

Fig. 6. Maximum deviation of the smaller peak between the true and the reconstructed distribution for the narrow and wide distribution as a function of the overall attenuation of the exciting laser beams. The intensity of the larger peaks was scaled to unity as a reference.

that skewing the profiles leads to apparently high local deviations. However, when measured signals are considered, it will reveal that the deviations shown in Fig. 5(a) for the low-attenuation case are insignificant within the noise level of a measurement.

The evaluation of the model distribution by use of Eq. (9) clearly shows errors that are due to the differentiation scheme. Deviations of $15 \%$ are observed, yet the overall structure of the assumed distribution has good recovery. A singularity in the center of the model profile occurs for the wide model function because of numerical reasons. In comparison with the $50 \%$ overall attenuation, the reconstruction for distributions that attenuate the light by $95 \%$ shows the limitations of the linear addition of the two signal contributions. For both model distributions, a substantial spatial distortion is noted, resulting in unacceptable reconstruction values, in particular for the wide distribution. The spatial shape of the narrow profile could be regarded as sufficient reconstruction; however, the peak intensity ratio is not recovered. The error of the peak intensity ratio when the reconstruction uses Eq. (4) is plotted in Fig. 6 as a function of the overall attenuation. The reconstruction based on Eq. (9) recovers the original distribution with reasonable accuracy in terms of spatial shape and peak ratio.

In view of experimental research, it is interesting to examine how much influence the unequal initial intensities for the forward and backward beams have. For both fuel distributions, the change of any of the initial intensities did not affect the ratio-based reconstruction method, even in cases in which one beam was an order of magnitude more intense. For the simple addition-based approach, the results are more diverse. In the case of the narrow fuel distribution, differences between initial intensities of a factor of 2 will result in deviations of as much as $20 \%$, up from $7 \%$ for equal-intensity beams. For the wide fuel distribution, the errors exceed $200 \%$.

For a successful recovery of the true fuel distribu- 


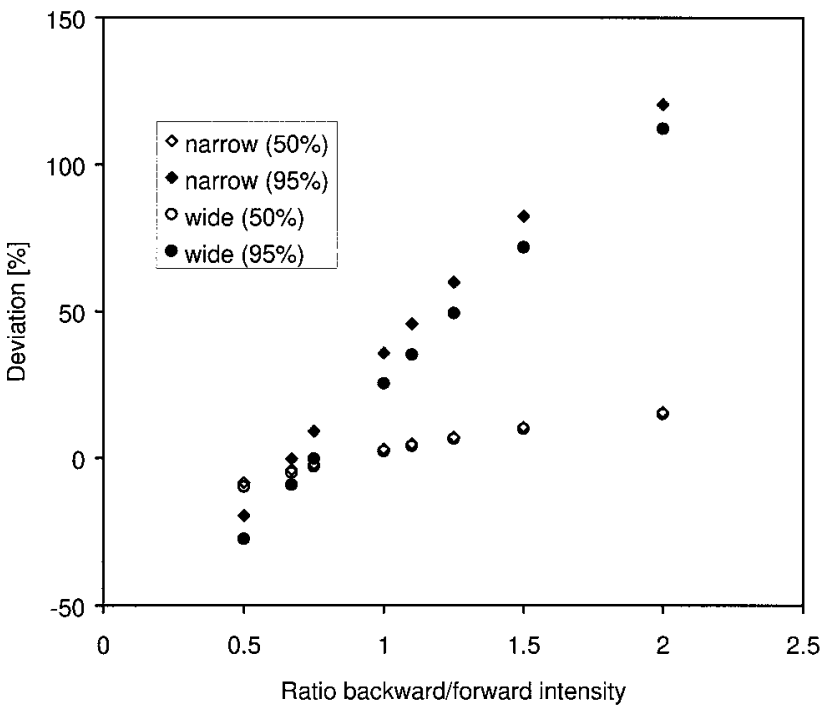

Fig. 7. Maximum deviation of the smaller peak between the true and the reconstructed distribution for the narrow and wide distribution as a function of the initial intensity ratio of backwardforward laser beams. The intensity of the larger peaks was scaled to unity as a reference.

tion in a spray by use of the counterpropagating illumination and detection of the signals from both beams simultaneously, the initial intensity of the beams should be identical as much as possible. The systematic error introduced by unequal initial intensities is shown for a peak intensity ratio in Fig. 7.

An example of Mie-scattering images of an impinging spray, obtained as an average of 15 individual exposures, is shown in Fig. 8. It should be noted that for illustration enhancement the gamma factor for the display was chosen to be 2.9. Separate images for forward and backward illumination were recorded and then added to analyze the overall attenuation. The overall attenuation across the spray should not exceed approximately $80 \%$ to keep systematic errors below 10\% (see Fig. 6). A simple check for the overall attenuation is to record averaged images with one excitation beam only and then to calculate the ratio of the two images. A maximum attenuation of $80 \%$ will result in a measured signal intensity ratio $I_{\text {forward }}$ to $I_{\text {backward }}$ of $100 / 20=5$ for the left-hand side of the image whereas the ratio will be $20 / 100=0.2$ for the right-hand side. If the ratio anywhere in the image is within these limits, the reconstruction by use of Eq. (4) will be successful. This is shown in the profile of Fig. 9.

Our attempts to reconstruct the fuel distribution using Eq. (10) from the measured individual images of Fig. 8 failed. When the differentiation according to Eq. (10) is used, the noise in the experimental data leads to negative values for recovered signal intensities. We did not obtain a satisfactory improvement by Gaussian filtering the data to remove noise. Unless better filtering schemes are applied, this method is not suitable to reconstruct the true fuel distribution, despite its superior theoretical quality.
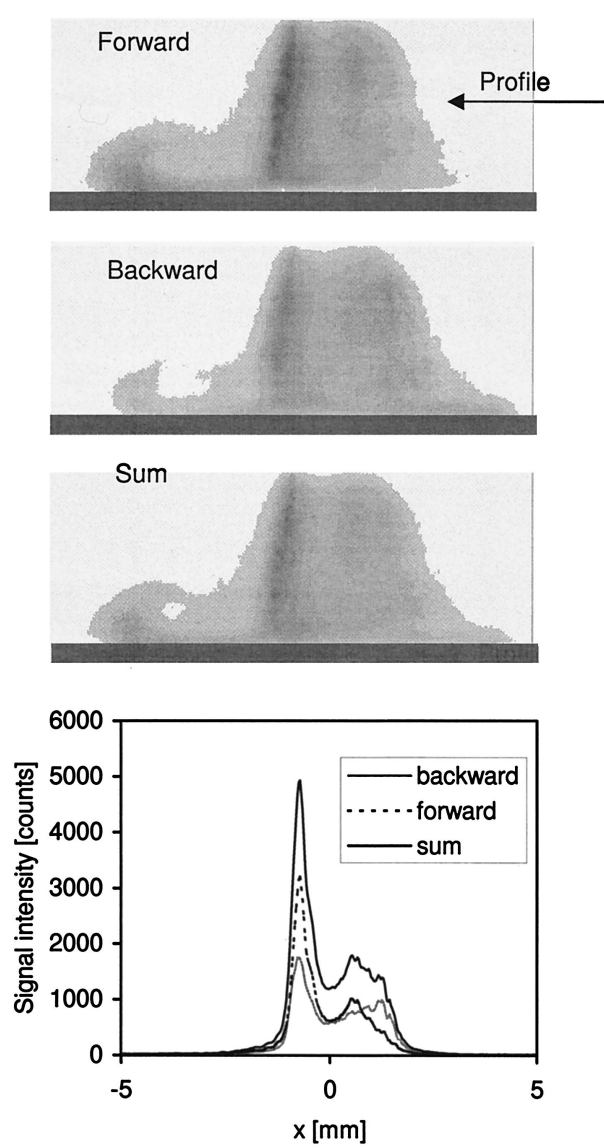

Fig. 8. Averaged Mie-scattering images of an impinging spray. A horizontal profile at the indicated location is extracted for numerical illustration.

\section{B. Signal Attenuation}

Attenuation of the LIF signals reaches $60 \%$ when the signals from the cuvette travel through the entire spray. In a single spray event, as shown in Fig. 10, an inverse top-hat profile is measured. This type of profile, and the information obtained from the corrected LIF images, shows that attenuation of the LIF signals occurs only in the outer regions of the spray where the droplet density is high. This means that LIF images measured with the exciting beam located

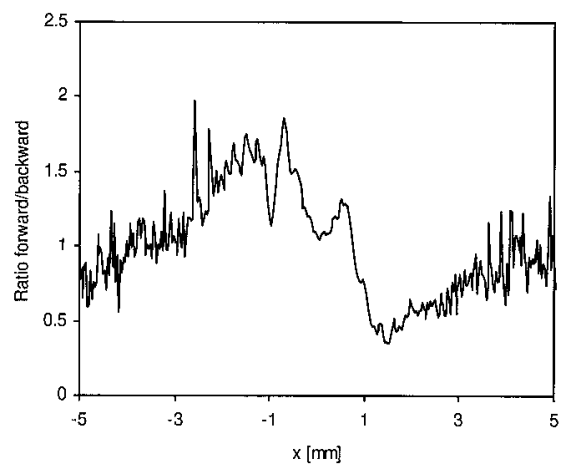

Fig. 9. Ratio of Mie-scattering signals obtained by forward and backward illumination only. The profile is calculated from the data of Fig. 8. 

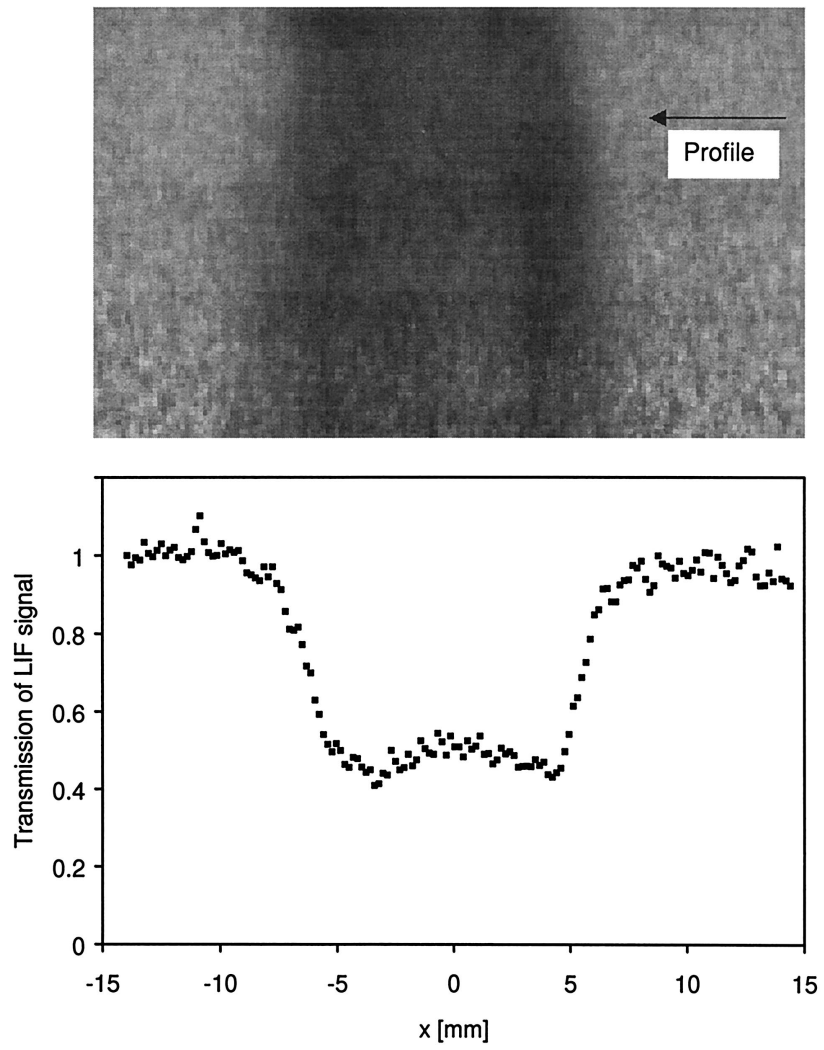

Fig. 10. Single-shot transmission measurement for LIF signals through a hollow-cone spray.

in the center of the spray will be measured with an attenuation of approximately $25-30 \%$, which does not change much spatially. This finding simplifies the reconstruction of spray measurements considerably. Caution should be applied for measurement of unknown sprays, as the spatial distribution of the fuel will not always be symmetric. The setup for the laser dye cuvette for the signal attenuation measurements is simple and quick. The performance of such a measurement, especially when there is an apparent strong deviation from a hollow-cone structure, is recommended. Ignoring the attenuation effects would lead to systematic errors of the order of $30 \%$ for absolute data. The relative error, however, as given by the deviation from a flat profile is considerably smaller.

\section{Conclusions}

The detailed investigation of attenuation effects in imaging experiments of hollow-cone sprays revealed that a simple bidirectional excitation setup could be used successfully to measure Mie scattering and LIF signals for a wide range of conditions. To keep systematic errors below 10\%, the overall attenuation of each beam should not exceed $80 \%$. The initial intensity ratio of forward and backward beams sensitively influences the measurements. Under these conditions a bidirectional illumination allows adequate imaging of hollow-cone sprays by use of a single
CCD camera only. Sequential measurement of signals for each exciting beam and processing the data with a different algorithm based on spatial derivatives could avoid the discussed restrictions. No dependence of the reconstructed images on initial laser intensities occurs when this approach is used. The method that extracts the true distribution by the gradient of the natural logarithm of the ratio of the measured signals has no restriction for the intensity or spatial distribution of the fuel. However, resolution effects are noted in particular when a simple differentiation scheme is used. Furthermore, noise from the measured images could not be removed sufficiently to allow the application of the gradientbased method. New filtering schemes, such as nonlinear anisotropic diffusion techniques, ${ }^{15}$ show promising results and will be tested for this application.

Analysis of the attenuation of fluorescence signals by sprays showed values of the overall attenuation to be similar to the attenuation of exciting laser beams. Although a single-shot image reconstruction for this effect seems difficult because of a lack of threedimensional fuel distribution data, information about averaged signal attenuation can be used to judge the estimated local error.

This research was sponsored by U.S. Department of Energy contracts DE-FG04-99AL66236 and DEFG02-98ER14915, subcontract 14412-S1, Division of Chemical Sciences, Office of Basic Energy Sciences, and the General Motors Corporation through the General Motors Satellite Research Laboratory at the University of Michigan. The authors also acknowledge the contributions of M. C. Drake.

\section{References}

1. F.-Q. Zhao, M.-C. Lai, and D. L. Harrington, "Automotive spark-ignited direct-injection gasoline engines," Prog. Energy Combust. Sci. 25, 437-562 (1999).

2. C. Schulz, V. Sick, J. Wolfrum, V. Drewes, M. Zahn, and R. Maly, "Quantitative 2D single-shot imaging of NO concentrations and temperatures in a transparent SI engine," in Proceedings of the Twenty-Sixth Symposium (International) on Combustion (Combustion Institute, Pittsburgh, Pa., 1996), pp. 2597-2604.

3. M. Knapp, A. Luczak, H. Schlüter, V. Beushausen, W. Hentschel, and P. Andresen, "Crank-angle-resolved laserinduced fluorescence imaging of $\mathrm{NO}$ in a spark-ignition engine at $248 \mathrm{~nm}$ and correlations to flame front propagation and pressure release," Appl. Opt. 35, 4009-4017 (1996).

4. F. Hildenbrand, C. Schulz, E. Wagner, and V. Sick, "Investigation of spatially resolved light absorption in a spark-ignition engine fueled with propane/air," Appl. Opt. 38, 1452-1458 (1999).

5. C. Preussner, C. Doering, S. Fehler, and S. Kampmann, "GDI: Interaction between mixture preparation, combustion system and injector performance," SAE paper 980498 (Society of Automotive Engineers, Warrendale, Pa., 1998).

6. J. Su, M. C. Drake, T. D. Fansler, and D. L. Harrington, "Towards quantitative characterization of transient fuel sprays using planar laser induced fluorescence imaging," presented at the Eleventh Annual Conference on Liquid Atomization and Spray Systems of the Institute for Liquid Atomization and 
Spray Systems, ILASS Americas '98, Sacramento, Calif., 17-20 May 1998.

7. H. M. Hertz and M. Alden, "Calibration of imaging laserinduced fluorescence measurements in highly absorbing flames," Appl. Phys. B 42, 97-102 (1987).

8. M. Versluis, N. Georgiev, L. Martinsson, M. Alden, and S. Kröll, "2-D absolute $\mathrm{OH}$ concentration profiles in atmospheric flames using planar LIF in a bi-directional laser beam configuration," Appl. Phys. B 65, 411-417 (1997).

9. D. G. Talley, J. F. Verdieck, S. W. Lee, V. G. McDonnell, and G. S. Samuelsen, "Accounting for laser sheet extinction in applying PLLIF to sprays," paper AIAA-96-0469, presented at the Thirty-Fourth Aerospace Sciences Meeting, Reno, Nev., 15-18 Jan. 1996 (American Institute of Aeronautics and Astronautics, New York, 1996).

10. A. Arnold, A. Buschmann, B. Cousyn, M. Decker, V. Sick, F. Vannobel, and J. Wolfrum, "Simultaneous imaging of fuel and hydroxyl radicals in an in-line four cylinder SI engine," SAE paper 932696 (Society of Automotive Engineers, Warrendale, Pa., 1993)

11. S. Einecke, C. Schulz, V. Sick, R. Schiessl, A. Dreizler, and U. Maas, "Two-dimensional temperature measurements in the compression stroke of a SI engine using two-line tracer LIF," SAE Trans. 107, 1060-1068 (1998)

12. J. M. Quagliaroli, G. Laufer, R. H. Kraus, and J. C. McDaniel, "Laser selection of $\mathrm{OH}$ fluorescence measurements in supersonic test facilities," AIAA J. 31, 520-527 (1993).

13. B. Stojkovic and V. Sick, "Spray evolution and impingement investigated with simultaneous Mie/LIF techniques," Appl. Phys. B (to be published).

14. M. Raffel, C. E. Willert, and J. Kompenhans, Particle Image Velocimetry: A Practical Guide (Springer-Verlag, New York, 1998).

15. J. Weickert, Anisotropic Diffusion in Image Processing (Teubner, Stuttgart, Germany, 1998). 